\title{
The ACE-inhibitor captopril improves myocardial perfusion in spontaneously diabetic (BB) rats
}

\author{
R. Rösen ${ }^{1}$, A.F. E. Rump ${ }^{1}$, P. Rösen ${ }^{2}$ \\ ${ }^{1}$ Institute of Pharmacology University of Cologne, Cologne, Germany \\ ${ }^{2}$ Diabetes Research Institute at the Heinrich-Heine-University, Düsseldorf, Germany
}

Summary The aim of this study was to examine the influence of inhibition on angiotensin converting enzyme (ACE) of myocardial function and perfusion of the rat impaired by diabetes. Spontaneously diabetic rats were treated with the ACE-inhibitor captopril for 4 months. Cardiac performance was analysed in the isolated heart perfused at constant volume. Epicardial perfusion was determined by measuring changes in epicardial fluorescence after injection of a bolus of fluoresceinisothiocyanate-dextrane (3 $\mathrm{kDa}$ ) as described previously. As compared to untreated diabetic controls, captopril prevented the increase of end diastolic pressure, coronary perfusion pressure and vascular resistance. The intravascular volume was enlarged and the epicardial perfusion rate increased in hearts of diabetic rats treated with captopril as compared to diabetic controls. Treat- ment of diabetic rats with the ACE-inhibitor captopril (1) increases the number of perfused capillaries, and (2) can partly prevent the development of cardiac dysfunction in diabetes. Together with morphological data demonstrating an inhibition of interstitial and perivascular fibrosis in hearts of diabetic rats treated with captopril, our data suggest that ACE-inhibition is cardioprotective in diabetes. These observations are also compatible with the assumption that an accelerated generation of angiotensin II may be involved in the pathophysiological chain of events leading to diabetic cardiopathy. [Diabetologia (1995) 38: 509-517]

Key words Diabetes mellitus, cardiopathy, ACE-inhibition, captopril, BB rats, regional perfusion, myocardial function.
The incidence of cardiovascular complications is severely increased in diabetes mellitus by the development of macrovascular complications. However, coronary heart disease can only partly explain the increased rate of cardiovascular complications in diabetes, since cardiac risk remains elevated even in the absence of atherosclerosis [1-3]. In patients, impairment of diastolic relaxation and signs of cardiac neu-

Received: 19 April 1994 and in revised form: 20 October 1994

Corresponding author: Professor Dr. P. Rösen, Diabetes-Forschungsinstitut, Auf'm Hennekamp 65, D-40225 Düsseldorf, Germany

Abbreviations: ACE, Angiotensin converting enzyme; CVR, coronary vascular resistance; EDP, end diastolic pressure; LVP, left ventricular pressure; CPP, coronary perfusion pressure. ropathy have been consistently reported [3, 4]. Additionally, cardiac performance is further impaired in diabetic patients by development of small vessel disease $[5,6]$. In animal models using chemically-induced forms of insulin deficiency or spontaneously diabetic rats and mice, the metabolic disturbances following insulin deficiency cause impairment of diastolic and systolic heart performance, which can be totally prevented by treatment of the animals with insulin [7-11]. The various forms of cardiac dysfunction in diabetes which develop independently from coronary heart disease, are often called "diabetic cardiomyopathy" [12-14].

The mechanisms underlying these complex disturbances of heart function in diabetes are not fully understood and several hypotheses have been suggested to link the metabolic disturbances observed in the diabetic heart with impairment of heart function [4, 
7, 10]. Microangiopathic changes [14-16], abnormalities in vascular reactivity $[15,17]$, altered autonomic function $[9,18]$, increased stiffness of the ventricular: wall and perivascular thickening, interstitial accumulation of connective tissue and matrix proteins $[4,9$, $18,19]$, and defects in the regulation of calcium homeostasis [20] have been claimed to contribute as aetiological factors to disturbances of heart function in diabetes.

We suggested recently [4] that the renin-angiotensin-system is activated in diabetes with increasing work load because of the limited capacity of the diabetic heart to supply oxygen and to generate energy in sufficient amounts. An enhanced generation of angiotensin II could explain some of the changes in cardiac performance observed in diabetes: activation of the sympathetic nervous system $[21,22]$, promotion of cardiac and vascular fibrosis resulting in increased myocardial stiffness and impaired myocardial contractility, and disturbances in the regulation of coronary flow [23-25]. Moreover, coronary vasoconstriction and myocardial necrosis have been reported to be caused by an enhanced generation of angiotensin II in other forms of heart failure [23, 24], which can contribute to a further restriction and impairment of cardiac function. In various forms of heart failure the use of angiotensin converting enzyme (ACE)-inhibitors is now an established therapy [23]. In clinical settings, ACE-inhibition has been shown to improve survival and clinical symptoms $[23,26]$. In experimental studies evidence has been presented that ACE-inhibition can inhibit the development of hypertrophy and fibrosis as well as improve endothelial function [27]. However, influence of ACE-inhibitors on the function of the diabetic heart has not yet been studied, although inhibition of ACE may enlarge the availability of bradykinin in diabetic hearts which has been shown to improve the insulin sensitivity and the conversion of glucose in hearts of diabetic rats and in skeletal muscle of patients [28-30].

To test the hypotheses that an enhanced generation of angiotensin II contributes largely to cardiac dysfunction as observed in diabetes, we treated diabetic rats with captopril as an ACE inhibitor in concentrations which do not interfere only with the peripheral angiotensin II formation, but presumably also with the local myocardial generation of angiotensin II. As a model of insulin-dependent diabetes mellitus (IDDM) we used the diabetic BB (Biobreeding) rat which is a close counterpart to human $\operatorname{IDDM}[4,10]$.

\section{Materials and methods}

Animals. Male BB rats were delivered by Møllegaard, Ejby, Denmark, at the age of 60 days. The animals were kept in the animal laboratory of the Diabetes-Forschungsinstitut under standard housing conditions. The animals had free access to water and received a standard diet (Sniff, Soest, Germany). At the age of $80-100$ days, $70 \%$ of the rats became spontaneously diabetic and had to be treated with insulin (Insulin super lente; Novo, Bagsvaerd., Denmark, 3-4 IU/day) according to the metabolic demand to prevent ketoacidosis and excessive hyperglycaemia [9]. The animals were controlled daily for glucosuria (Gluketur sticks; Boehringer Mannheim, Mannheim, Germany), weekly for weight and every 4 weeks for blood glucose by the hexokinase method using a glucose analyser (Beckman, Munich, Germany). As controls, diabetes-resistant rats were used, which develop diabetes at an incidence of less than $1 \%$. Just after killing, blood was taken for the determination of plasma insulin using a commercial RIA-kit (Pharmacia, Freiburg, Germany) with rat insulin (Novo) as standard and glycated haemoglobins by affinity chromatography (Isolab, Munich, Germany).

Sodium, potassium and haematocrit were determined by standard laboratory techniques, triglycerides by the glyceroloxidase method according to the instructions of the manufacturer (Boehringer Mannheim) and ACE-activity according to Cushman and Cheung [31].

After verification of the diabetic state, one group of animals was treated with captopril for 4 months. Captopril was supplied in the drinking water. Since the amount of drinking water was different $(18 \mathrm{ml} /$ day in controls and $112 \mathrm{ml} /$ day for diabetic rats), the amount of drinking water was regularly controlled and the concentration of captopril adjusted to ensure an uptake of $30 \mathrm{mg} / \mathrm{kg}$ body weight and day. The plasma concentration of sodium, potassium and the haematocrit were routinely checked every month. Each group of rats consisted of at least five to eight animals.

Heart function in vivo. Heart function was determined in anaesthetised rats (Nembutal, $30 \mathrm{mg} / \mathrm{kg}$ body weight) after inserting a Millar tip catheter into the carotid artery as described previously [8].

Isolated perfused heart. Additionally, hearts were isolated and perfused according to Langendorff with Krebs-Henseleit-bicarbonate buffer $\left(\mathrm{Na}^{+} 143, \mathrm{~K}^{+} 5.9, \mathrm{Ca}^{2+} 2.5, \mathrm{Mg}^{2+} 1.18, \mathrm{Cl}^{-}\right.$ 127.7, $\mathrm{HCO}_{3}^{-} 25, \mathrm{H}_{2} \mathrm{PO}_{4}^{-1} 1.18$, sulfate 1.18 and glucose 10.1 $(\mathrm{mmol} / \mathrm{l}), \mathrm{pH} 7.4)$ saturated with $5 \% \mathrm{CO}_{2}-95 \% \mathrm{O}_{2}$ as previously published [17].

There was no ACE-inhibitor applied during heart perfusion. Global coronary flow was kept constant at 5.0, 10.0, and $13.0 \mathrm{ml} / \mathrm{min}$ with a roller pump. Hearts were allowed to beat spontaneously. End systolic and end diastolic left ventricular pressure were measured via an intraventricular catheter. Regional perfusion parameters on the left ventricular epicardium were analysed from indicator elution kinetics registered by surface fluorescence after bolus injection of fluoresceinisothiocyanate-dextrane (MG $3 \mathrm{kDa}, 100 \mu \mathrm{l}$ ) as described previously $[17,32]$. The elution profiles could be attributed to two components of regional perfusion: a fast, vascular and a slow, extravascular component with half-lives in the range of seconds and minutes, respectively. It has been shown previously that the slow component was absent when fluoresceinisothiocyanate-dextrane of high molecular weight $(70 \mathrm{kDa})$ was used [17]. The maximal signal height after bolus injection was proportional to the intravascular volume, whereas the extravascular indicator concentration could be extrapolated from half-log plots of the slow component and corresponded to the transcapillary permeability. The half-lives of the elution kinetics corresponded to the intraluminal flow rate (vascular perfusion rate, $T / 2$ in seconds) and to the interstitial-extravascular exchange rate (extravascular clearance, $t / 2$ in minutes). 
Table 1. Influence of ACE-inhibition by captopril on the biochemical properties of diabetic BB rats

\begin{tabular}{lccc}
\hline Parameter & Controls & Diabetes & $\begin{array}{l}\text { Diabetics } \\
\text { treated with } \\
\text { captopril }\end{array}$ \\
\hline Body weight $(\mathrm{g})$ & $462 \pm 15$ & $426 \pm 19$ & $422 \pm 7^{\mathrm{a}}$ \\
$\begin{array}{l}\text { Blood glucose } \\
\left(\mathrm{mmol}^{\prime} \mathrm{l}\right)\end{array}$ & $7.3 \pm 1.5$ & $15.6 \pm 1.5^{\mathrm{a}}$ & $16.5 \pm 1.3^{\mathrm{a}}$ \\
$\mathrm{HbA}_{1}(\%)$ & $5.7 \pm 0.5$ & $10.7 \pm 1.4^{\mathrm{a}}$ & $8.5 \pm 0.9^{\mathrm{a}}$ \\
$\mathrm{ACE}(\mathrm{U} / \mathrm{l})$ & $60.3 \pm 8.2$ & $98.9 \pm 8.5^{\mathrm{a}}$ & $\mathrm{n.d} .^{\mathrm{b}}$ \\
Sodium (meq/1) & $142 \pm 2$ & $139 \pm 2$ & $140 \pm 3$ \\
Potassium (meq/1) & $6.6 \pm 0.4$ & $7.5 \pm 0.5$ & $7.2 \pm 0.4$ \\
Haematocrit $(\%)$ & $42.2 \pm 2.3$ & $41.5 \pm 1.3$ & $42.0 \pm 1.8$ \\
\hline
\end{tabular}

After manifestation of diabetes, the diabetic BB rats were treated with insulin. One group of rats additionally received captopril $\left(30 \mathrm{mg} \cdot \mathrm{kg} \mathrm{b}\right.$. w. $\left.{ }^{-1} \cdot \mathrm{day}^{-1}\right)$. After a diabetes duration of 4 months the given parameters were determined as described in Methods. The data are given as mean \pm SEM, $n=5-8$ animals per group, n.d. not detectable.

${ }^{a} p<0.05$ statistically significant difference between controls and diabetic rats;

${ }^{\mathrm{b}} p<0.05$ statistically significant difference between untreated and treated diabetic rats

\section{Statistical analysis}

Data are given as mean \pm SEM. In any experimental group five to eight animals were studied. Differences between groups were analysed by analysis of variance (ANOVA), differences between means by the Scheffé test, assuming a statistically significant difference at $p<0.05$ and a normal distribution of data.

\section{Results}

Despite daily administration of insulin, the diabetic $\mathrm{BB}$ rats were hyperglycaemic as ascertained from the elevated postprandial blood glucose levels and the increased amounts of glycated haemoglobin. Triglycerides ranged between 3.5 and $8.5 \mathrm{mmol} / 1$. Glucosuria, ketonuria, and polydipsia were additionally typical symptoms found in the diabetic BB rats. Cap- topril had no significant influence on the body weight, the blood glucose, and plasma insulin, but reduced the level of glycated haemoglobin. The concentrations of sodium and potassium in plasma were not significantly affected by the diabetic state or by treatment with captopril (Table 1). The haematocrit was not significantly different between the experimental groups $(42 \pm 2,41 \pm 3$ and $39.5 \pm 2.5 \%$ in control, diabetic and captopril-treated diabetic rats, respectively).

The activity of angiotensin converting enzyme was elevated in the serum of diabetic rats as compared to controls $(60.3 \pm 8.2$ vs $98.9 \pm 8.5 \mathrm{U} / 1, p<0.05)$, but not be detectable in serum of captopril-treated diabetic or control rats.

Heart function in vivo. As already published [9], cardiac performance was reduced in the diabetic $\mathrm{BB}$ rat: heart rate, the maximal systolic pressure in the left ventricle, and the diastolic relaxation velocity were reduced, whereas the velocity of contraction was unchanged by the development of the diabetic state.

Treatment of the animals with captopril had no effect on hearts of control, healthy rats. In diabetic animals, the left ventricular pressure, and the systolic aortic pressure were slightly reduced, the end diastolic pressure significantly reduced in the captopril group as compared to untreated diabetic BB rats (Table 2). The maximum of contraction and relaxation were not influenced by the treatment with captopril. The end diastolic pressure was significantly reduced in the captopril group as compared to untreated diabetic BB rats (Table 2).

Performance of the isolated perfused heart. Treatment with captopril had no significant influence on performance of the isolated heart of control, healthy rats perfused at constant volume (data not shown). Heart rate was not different between controls and diabetic rats and was not influenced by the coronary flow or treatment with captopril. With increasing coronary flow left ventricular pressure (LVP) rose sharply in the hearts of controls, but only slightly in those of un-

Table 2. Influence of ACE-inhibition by captopril on heart performance of diabetic BB rats in vivo

\begin{tabular}{lccc}
\hline Parameter & Controls & Diabetes & Diabetics treated with captopril \\
\hline Heart rate (beats/min) & $386 \pm 14$ & $348 \pm 12^{\mathrm{a}}$ & $361 \pm 22$ \\
Left ventricular systolic pressure $(\mathrm{mm} \mathrm{Hg})$ & $133 \pm 13$ & $115 \pm 3^{\mathrm{a}}$ & $101 \pm 8^{\mathrm{a}}$ \\
End diastolic pressure $(\mathrm{mm} \mathrm{Hg})$ & $0.5 \pm 0.2$ & $2.3 \pm 0.4^{\mathrm{a}}$ & $1.1 \pm 0.2^{\mathrm{b}}$ \\
Maximal rate of contraction $(\mathrm{mm} \mathrm{Hg} / \mathrm{s})$ & $6740 \pm 791$ & $6965 \pm 386$ & $6767 \pm 344$ \\
Maximal rate of relaxation $(\mathrm{mm} \mathrm{Hg} / \mathrm{s})$ & $6943 \pm 673$ & $5961 \pm 357^{\mathrm{a}}$ & $5367 \pm 819^{\mathrm{a}}$ \\
Systolic aortic pressure & $104 \pm 3.5$ & $105 \pm 4.9$ & $98 \pm 7.3$ \\
\hline
\end{tabular}

Cardiac performance was determined in diabetic BB-rats, one group of which was additionally treated with captopril $\left(30 \mathrm{mg} \cdot \mathrm{kg} \mathrm{b.w} .^{-1} \cdot\right.$ day $\left.^{-1}\right)$. After a duration of diabetes for 4 months a Milar tip catheter was inserted into the carotid artery and heart performance determined as described. Data are given as mean \pm SEM, $n=5-8$ rats per group.

${ }^{a} p<0.05$ statistically significant difference between controls and diabetic rats;

${ }^{\mathrm{b}} p<0.05$ statistically significant difference between untreated and treated diabetic rats 


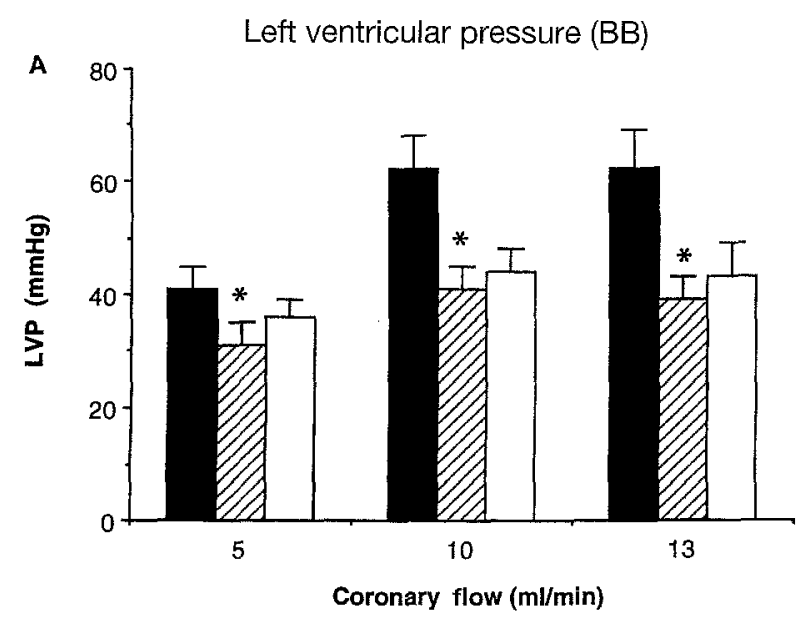

End diastolic pressure (BB)

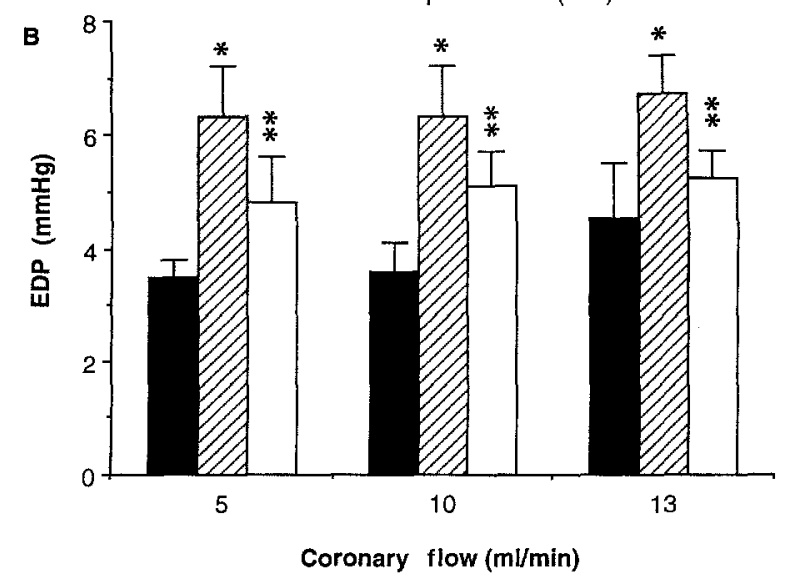

Fig. 1A, B. Influence of diabetes and captopril on (A) left ventricular (LVP) and (B) and end diastolic pressure (EDP) of Langendorff-perfused rat hearts at different flow rates. Hearts of captopril-treated and untreated diabetic BB and control rats were isolated and perfused at constant volume. The duration of diabetes and treatment with captopril was 4 months. Captopril did not influence the LVP and EDP in hearts of control rats. Data are given as mean \pm SEM, $n=5-8$ animals per group. ${ }^{*} p<0.05$ statistically significant difference between controls and untreated diabetic rats; ** $p<0.05$ statistically significant difference between untreated and treated diabetic rats. non-diabetic controls; $\square$ diabetics; $\square$ diabetics treated with captopril

treated and captopril-treated diabetic BB rats. Independently from coronary flow LVP was reduced in diabetes as compared to controls and not significantly influenced by captopril (Fig.1A). Thus, pressure $\times$ heart rate index was reduced in diabetes as compared to non-diabetic controls, and not changed by the treatment with the ACE-inhibitor.

The end diastolic pressure (EDP) was elevated in hearts of diabetic rats as compared to controls independently from the coronary flow. Treatment with captopril did not influence EDP in controls, but reduced the elevated EDP significantly in diabetes. A
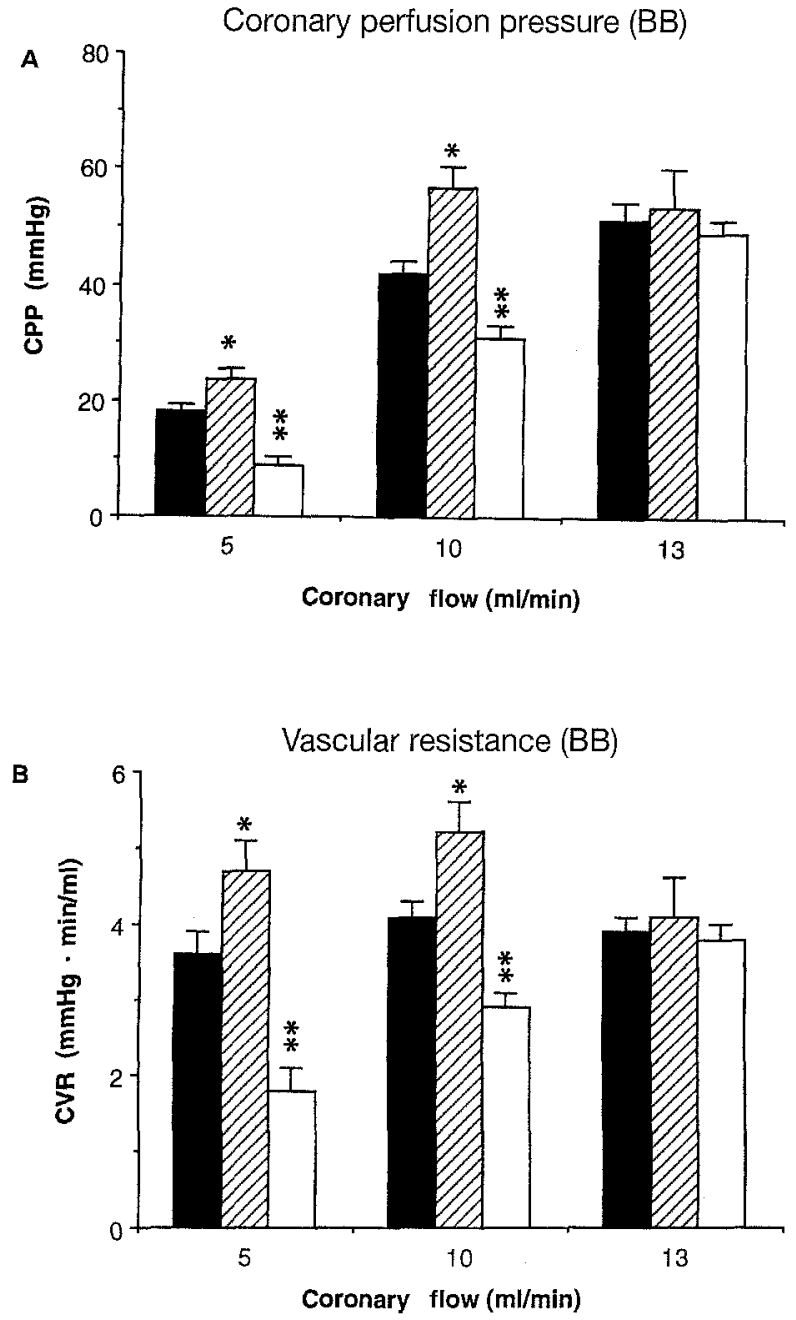

Fig. 2A, B. Influence of diabetes and captopril on (A) the coronary perfusion pressure (CPP) and (B) coronary vascular resistance (CVR) of Langendorff-perfused rat hearts at different flow rates. Hearts of captopril-treated and untreated diabetic $\mathrm{BB}$ and control rats were isolated and perfused at constant volume. The duration of diabetes and treatment with captopril was 4 months. Captopril did not influence the EDP and CPP in hearts of control rats. Data are given as mean \pm SEM, $n=5-8$ animals per group. ${ }^{*} p<0.05$ statistically significant difference between controls and untreated diabetic rats; $* * p<0.05$ statistically significant difference between untreated and treated diabetic rats. $\quad$ Non-diabetic controls; $\square$ diabetics; $\square$ diabetics treated with captopril

normalisation of this parameter was, however, not reached by the captopril treatment (Fig. 1B).

At low flow, coronary perfusion pressure (CPP) was different in hearts of control and diabetic $B B$ rats. In diabetes, CPP increased, however, more steeply with increasing coronary flow than in controls, but reached the same level at the highest flow rates $(13 \mathrm{ml} / \mathrm{min})$. In the hearts of captopril-treated diabetic rats CPP was significantly reduced and increased strictly depending on coronary flow (Fig. $2 \mathrm{~A}$ ).

Coronary vascular resistance (CVR) was nearly independent from coronary flow in control hearts. In 

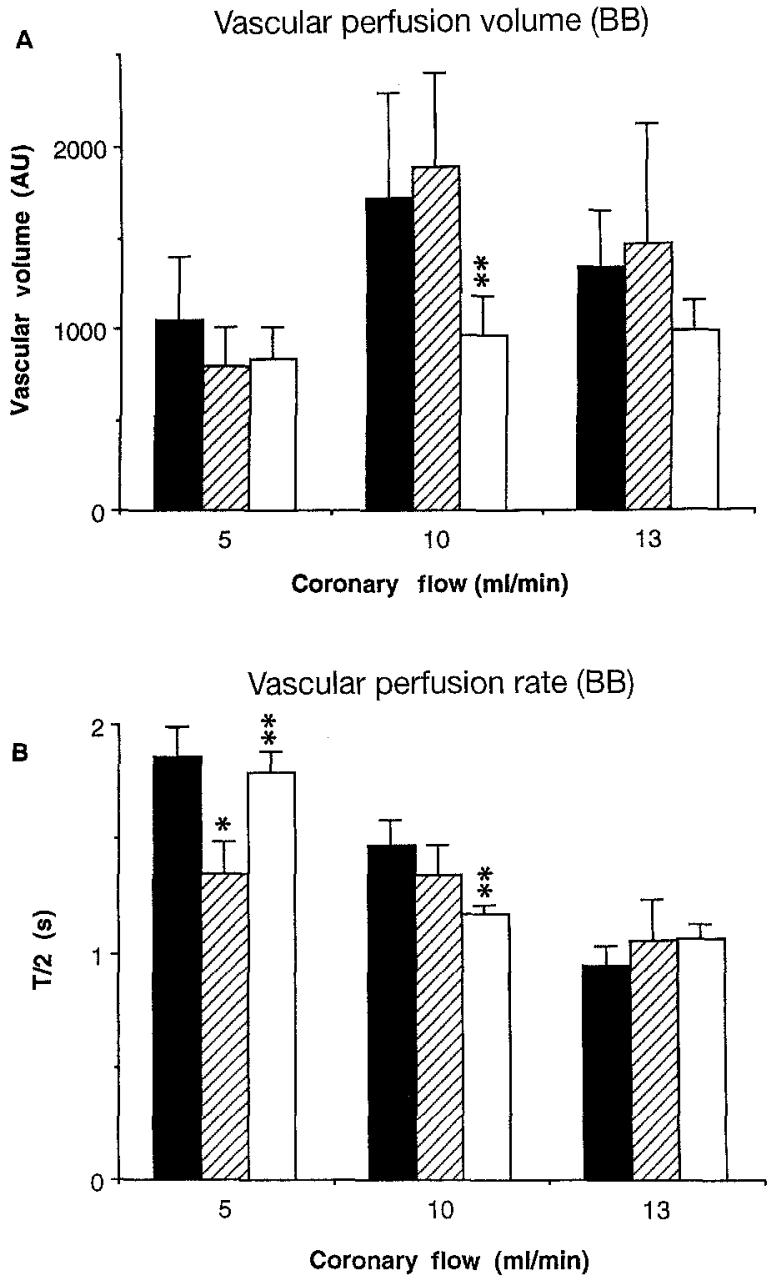

Fig. $3 \mathrm{~A}$, B. Influence of diabetes and captopril on vascular perfusion parameters of the left epicardium in Langendorffperfused rat hearts at different flow rates. Hearts of captopriltreated and untreated diabetic BB rats and controls were isolated and perfused at constant volume. The duration of diabetes and of treatment with captopril was 4 months. Vascular perfusion volume (A) and intravascular elution half-life (B) of fluorescence indicator wash-out was analysed at different coronary flow rates after bolus injection of fluoresceinisothiocyanate-dextrane $(3 \mathrm{kDa})$ by determination of the time course of epicardial fluorescence as described previously [17]. Data are given as mean $\pm \mathrm{SEM}, n=5-8$ animals per group. $* p<0.05$ statistically significant difference between controls and untreated diabetic rats; $* * p<0.05$ statistically significant difference between untreated and treated diabetic rats. Non-diabetic controls; $\exists$ diabetics; $\square$ diabetics treated with captopril

diabetes CVR was significantly increased at flow rates of 5 and $10 \mathrm{ml} / \mathrm{min}$ as compared to controls. Treatment of diabetic rats with captopril reduced CVR to about $2 \mathrm{~mm} \mathrm{Hg} / \mathrm{ml}$. In contrast to controls and diabetic hearts, a gradual increase of CVR was observed in hearts of diabetic BB rats treated with captopril dependently from coronary flow. At the highest coronary flow studied CVR was not any longer different in the three experimental groups (Fig. 2B).
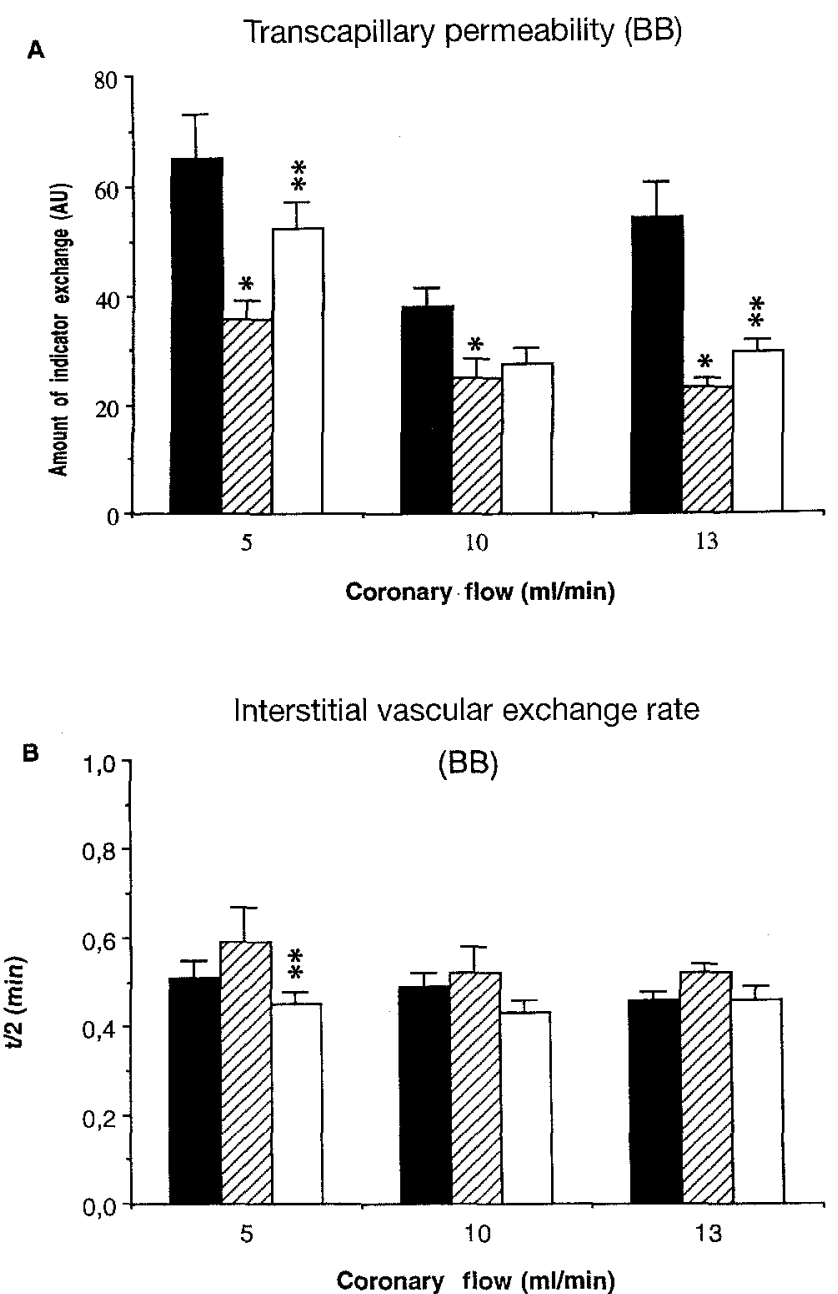

Fig. 4A, B. Influence of diabetes and captopril on extravascular perfusion parameters of Langendorff-perfused rat hearts at different coronary flow rates. Hearts of captopril-treated and untreated diabetic BB rats and controls were isolated and perfused at constant volume. The duration of diabetes and of treatment with captopril was 4 months. Permeability of fluoresceinisothiocyanate-dextrane (A) was determined by the amount of indicator in the interstitium after bolus transit. (B) Half-life of interstitial-vascular indicator exchange was measured by analysing the slow component of the time course of epicardial fluorescence. Further methodological details are described in [17]. Data are given as mean \pm SEM, $n=5-8$ animals per group. $* p<0.05$ statistically significant difference between controls and untreated diabetic rats; $* * p<0.05$ statistically significant difference between untreated and treated diabetic rats. $\square$ Non-diabetic controls; $\square$ diabetics; $\square$ diabetics treated with captopril

In controls, the local vascular perfusion rate was accelerated in parallel to the global coronary flow. The intravascular volume, on the other hand, increased only if the coronary flow was increased from 5 to $10 \mathrm{ml} / \mathrm{min}$ and declined at very high flow rates. Thus, the increase in global coronary flow caused a regionally accelerated flow rate and the coronary vessels were only dilated at the low flow rates, but not at high flow rates (overperfusion). As compared to controls the rate of local vascular perfusion was initially 
Table 3. Influence of ACE-inhibition by captopril on performance of the isolated heart perfused at constant flow of $10 \mathrm{ml} / \mathrm{min}$

\begin{tabular}{lccc}
\hline Parameter & Controls & Diabetics & $\begin{array}{l}\text { Treated } \\
\text { diabetics }\end{array}$ \\
\hline $\begin{array}{l}\text { Heart rate } \\
\text { (beats/min) }\end{array}$ & $204 \pm 21$ & $190 \pm 16$ & $193 \pm 7$ \\
$\begin{array}{l}\text { Left ventricular } \\
\text { pressure (mm Hg) }\end{array}$ & $62 \pm 4$ & $41 \pm 4^{\mathrm{a}}$ & $44 \pm 4$ \\
$\begin{array}{l}\text { End diastolic } \\
\text { pressure (mm Hg) }\end{array}$ & $3.6 \pm 0.5$ & $6.3 \pm 0.9^{\mathrm{a}}$ & $5.1 \pm 0.6^{\mathrm{b}}$ \\
$\begin{array}{l}\text { Coronary perfusion } \\
\text { pressure (mm Hg) }\end{array}$ & $42.0 \pm 2.0$ & $52.6 \pm 3.8^{\mathrm{a}}$ & $31.0 \pm 2.2^{\mathrm{b}}$ \\
$\begin{array}{l}\text { Coronary vascular } \\
\text { resistance (mm Hg/ml) }\end{array}$ & $4.1 \pm 0.2$ & $5.2 \pm 0.4^{\mathrm{a}}$ & $2.9 \pm 0.2^{\mathrm{b}}$ \\
T/2 (s) & $1.47 \pm 0.11$ & $1.34 \pm 0.13$ & $1.17 \pm 0.03^{\mathrm{b}}$ \\
t/2 (min) & $0.49 \pm 0.03$ & $0.52 \pm 0.06$ & $0.43 \pm 0.03^{\mathrm{b}}$ \\
$\mathrm{e}^{\mathrm{A}}$ (A. U.) & $724 \pm 564$ & $1888 \pm 503^{\mathrm{a}}$ & $963 \pm 214^{\mathrm{b}}$ \\
$\mathrm{e}^{\mathrm{a}}$ (A.U.) & $38.0 \pm 3.4$ & $24.9 \pm 3.7^{\mathrm{a}}$ & $27.6 \pm 2.6$ \\
\hline
\end{tabular}

After a diabetes duration of 4 months, hearts of control and diabetic BB-rats were isolated and perfused at constant perfusion volume. Cardiac performance was determined as described in Methods. Groups of control and diabetic rats were additionally treated with captopril $\left(30 \mathrm{mg} \cdot \mathrm{kg} \mathrm{b}\right.$..$^{-1} \cdot$ day $\left.^{-1}\right)$. Captopril had no significant influence on cardiac performance of control rats. Data are given as mean \pm SEM, $n=5-8$ rats per group.

$\mathrm{T} / 2$, Half time of the fast elution kinetics = vascular perfusion rate;

$t / 2$, half time of the slow elution kinetics $=$ interstitial-extravascular exchange rate;

$\mathrm{e}^{\mathrm{A}}$, intravascular volume; $\mathrm{e}^{\mathrm{a}}$, transcapillary permeability; $\mathrm{AU}$, arbitrary units;

${ }^{a} p<0.05$ statistically significant difference between controls and untreated diabetic rats;

${ }^{\mathrm{b}} p<0.05$ statistically significant difference between untreated and treated diabetic rats

higher in diabetic hearts $(5 \mathrm{ml} / \mathrm{min})$, but not different at higher coronary flow rates (10 and $13 \mathrm{ml} / \mathrm{min})$. The intravascular volume was the same in both kinds of hearts (Fig.3). Treatment of diabetic BB rats with captopril normalised the initially regional elevated perfusion rate $(5 \mathrm{ml} / \mathrm{min})$. Increasing the coronary flow, however, caused an acceleration of the local vascular perfusion rate. In contrast to hearts of controls and untreated diabetic rats, the intravascular volume was not influenced in hearts of captopril-treated diabetic hearts by changes in the global coronary flow (Table 3, Fig. 4).

The smaller the regional vascular perfusion volume, the smaller is the luminal surface for the exchange of the indicator across the vessel wall. The more accelerated the vascular transit (perfusion rate), the shorter is the exposition time of the vascular surface to the indicator. Both factors caused a significant diminution of permeability in the hearts of the diabetic BB rats (Fig. 4A), similarly as previous- ly reported for streptozotocin diabetic rats [17], although the driving force of the exchange (CVR) was enhanced. Treatment with captopril increased the transcapillary permeability at low flow rates.

\section{Discussion}

Previously we and others have shown that the diabetic BB rat is a reliable model for IDDM and that a distinct form of cardiopathy rapidly develops in these hyperglycaemic and insulin-treated rats affecting the vasculature, the cardiac nervous systems and the muscle cells $[4,9,10]$. We hypothesised that the renin-angiotensin system becomes activated in diabetes and that an enhanced formation or action of angiotensin II significantly contributes to the development of diabetic cardiopathy. The results of this study at least partly confirm the hypothesis that the renin-angiotensin system is activated as follows from the increased plasma ACE-activity in BB rats. A similar increase has been observed by others in experimental studies using the streptozotocin diabetic rats $[33,34]$. An augmented plasma ACE-activity has also been determined in IDDM patients with microangiopathy [35]. We also show that treatment of diabetic rats with the ACE-inhibitor captopril exerts distinct, protective effects on myocardial perfusion of the diabetic heart and can partly prevent the development of vascular and nervous cardiac dysfunction in diabetes.

The EDP was clearly reduced by treatment of diabetic rats with captopril in both experimental models. This diminution of EDP can be taken as an indicator of an improved relaxation of the diabetic heart. Since the velocity of relaxation delayed in diabetes was not influenced by captopril and since the diabetes-induced increase in EDP largely reflects the increased stiffness of ventricles in diabetes by accumulation of collagen and other matrix proteins $[4,9,19$, $25]$, we assume that the diminution of the end diastolic pressure reflects changes in the texture of cardiac vasculature and wall, but not an effect of the ACE inhibitor on the electric properties of the heart. In line with this assumption it has been shown that ACE inhibitors inhibit the deposition of collagen and other matrix proteins and thus can prevent the development of interstitial fibrosis [25]. In diabetic hearts, we showed by morphological techniques that the development of interstitial and perivascular fibrosis is significantly inhibited in diabetic BB rats by ACE inhibition [36]. That captopril can directly alter the diastolic-pressure relationship has also been demonstrated previously by Raya et al.[37]. In agreement with these data, a close relationship between the interstitial fibrosis and the activity of the renin-angiotensin system has been observed in humans. Treatment with ACE inhibitors did not only result in a re- 
duction of blood pressure, but also normalised impaired diastolic filling in the left ventricle [26]. On the other hand, it has to be considered that the reduced left ventricular stiffness might also be associated with a reduced end diastolic volume, which could contribute to the beneficial effect described.

Heart rate, left ventricular pressure, and contraction and relaxation velocities were not significantly altered by treatment of control and diabetic rats with captopril. It has been consistently reported that the heart rate is reduced in the perfused heart of diabetic rats $[8,10,38]$. Since we did not observe a similar alteration in vivo, the reduced heart rate observed in an isolated heart preparation indicates a specific defect in the sympathetic cardiac nerves [9], which is compensated in vivo by central nervous mechanisms. In humans and rats a neuropathy of the heart develops in diabetes, but it seems that the cardiac sympathetic nerves are affected more severely and earlier in the rat as compared to humans and other species $[9,39,40]$. In healthy control rats we did not observe any effect of the used dosage of captopril on heart performance at all, presumably because the activity of the ACE inhibitor is balanced by counterregulatory mechanisms to maintain blood pressure and LVP. In diabetes, the systolic aortic pressure was only slightly reduced by the treatment with captopril. Our data suggest furthermore that angiotensin independent mechanisms seem to be relevant for changes in the velocities of contraction and relaxation observed in diabetic rat hearts $[7,8,10,38,41]$.

In contrast to humans [42] the dosage of $30 \mathrm{mg}$. $\mathrm{kg}^{-1} \cdot$ day $^{-1}$ is well-tolerated in rats in agreement with observations of others [33] and does not lead to disturbances in the regulation of systemic blood pressure and myocardial mechanics. For extrapolation of the observations reported for humans, it has to be taken into account that the rat is obviously less sensitive to ACE inhibition than humans, since all effects of captopril were achieved by drug concentrations which are very high for humans, but did not significantly reduce the blood pressure in rats. Furthermore, in rats the conversion of angiotensin I to II can be totally inhibited by captopril [43], whereas in human myocardium angiotensin has been suggested to be mainly metabolised by a chymase activity which is insensitive to captopril [43].

A closer analysis of the effect of captopril on cardiac performance was performed using the isolated heart perfused at constant volume to enable the measurement of the time courses of epicardial fluorescence changes after injection of a bolus of fluoresceinisothiocyanate-dextrane. By this technique, the local perfusion rate and the local perfusion volume at the epicardium as well as the transcapillary exchange rates can be determined as discussed previously [17]. Several lines of evidence suggest that the autoregulation of coronary flow is disturbed in dia- betes and can be significantly improved by treatment of diabetic rats with captopril. Firstly, coronary perfusion pressure and coronary vascular resistance elevated in diabetes are significantly reduced by treatment with captopril. Secondly, the intravascular volume which remained nearly constant at increasing global coronary flow in hearts of captopril-treated diabetic $\mathrm{BB}$ rats, as well as the alterations in the vascular perfusion rate suggest that treatment with captopril alters the kinetics of the indicator elution. We interpret these observations as an indication of an increased number of perfused capillaries. This interpretation of our results is in line with morphological data showing that the mean capillary diameter is enlarged by about $25 \%$ in the diabetic heart at spontaneous coronary flow ( 8 to $9 \mathrm{ml} / \mathrm{min}$ ) as compared with controls, but that the density of capillaries in the left ventricle of diabetic BB rats is reduced [9]. Treatment with captopril causes a reduction in diameter of myocardial capillaries without changing the spontaneous coronary flow, but increases the density of myocardial capillaries [36]. Such an angiogenic action of captopril and other ACE-inhibitors has been demonstrated by others [27]. Thirdly, captopril enhances the permeability and partly normalises the disturbed permeability properties of epicardial vessels. The indicator permeation and the transcapillary exchange rates were reduced in hearts of diabetic $\mathrm{BB}$ rats indicating an increased tightness of coronary epicardial vessels in diabetes. These findings are in good agreement with earlier observations of hearts of streptozotocin diabetic rats [17]. It has to be mentioned, however, that the used indicator dilution technique represents a dynamic approach and that the local permeability of epicardial coronary vessels is determined by this method. With our technique we observe a local event at the epicardial surface during passage of the indicator bolus through the vessel lumen and do not measure the global exchange of indicator across the total myocardial vessel surfaces. These results cannot be extrapolated to the subepicardial vessels and the whole myocardial wall. Therefore our results are not comparable with data obtained for the whole heart by the indicator dilution technique [44], by which the distribution of the indicator is measured after complete equilibration and the exchange of indicator mostly occurs in the postcapillary venules of the heart. In any case, our findings indicate that despite the absence of apparent morphological changes in endothelium $[9,38]$ the function of myocardial endothelium is altered in diabetes and that at least the changes in permeability and tightness as measured by this technique can be prevented by ACE inhibition. It remains to be proved whether other early endothelial dysfunctions in diabetes such as the disturbed synthesis and release of the endothelial mediators prostacyclin and nitric oxide [45] can also be prevented by ACE inhibition. 
Together with the morphological data recently published [36] our observations clearly suggest that inhibition of ACE is cardioprotective in diabetes with respect to the myocardial texture and coronary perfusion system. Both effects are important in improving myocardial relaxation and perfusion of the heart in diabetes.

From these studies we can conclude that activation of ACE may play an important role in the development of cardiac dysfunction in diabetes. This conclusion is in line with other studies demonstrating cardioprotective effects of ACE inhibition in other experimental and clinical settings $[22,25,27$, 46]. Two different mechanisms can contribute to the observed cardioprotection: inhibition of the generation of angiotensin II or the delayed degradation of bradykinin. Angiotensin II has been shown to exert vasoconstriction, to stimulate growth of fibroblasts and deposition of collagen and other matrix proteins, to induce growth of myocardium $[25,46]$ and has numerous further actions on the central and peripheral nervous tissue $[47,21]$. It is therefore obvious that an accelerated generation of angiotensin II greatly influences the function and structure of myocardium and that inhibition of an enhanced formation of angiotensin can explain many of the observed alterations in function and structure of the heart caused by the diabetic state. Additionally or alternatively, our results could be explained by a delayed degradation of bradykinin. Bradykinin has been shown to increase the nutritional flow and the permeability of coronary vasculature leading to an improved local myocardial perfusion $[17,19,30]$. Furthermore, it has been shown that ACE inhibition and delayed degradation of bradykinin can prevent endothelial dysfunction induced by a variety of pathophysiological states $[27,48]$. Thus, there is some probability that cardioprotection by ACE inhibition is indirectly exerted by improvement of myocardial perfusion and prevention of hypoxic or ischaemic episodes. Additionally some evidence has accumulated that ACE inhibition and bradykinin accelerate the uptake and conversion of glucose in skeletal muscle and heart [28, 29]. A better provision of the heart with glucose and oxygen might help economise myocardial metabolism and to prevent an energy deficit because of the inability of the diabetic heart to adapt the glucose metabolism to the metabolic need $[8,28]$. Since there is no evidence in the rat of a specific myocardial ACE activity such as the chymase in human heart [43], we cannot distinguish between the two mechanisms and decide to which degree both mechanisms are involved in the cardioprotective effect of ACE inhibition by captopril.

In summary, our data confirm the initial hypothesis that the myocardial ACE activity may play an important pathophysiological role in the development of myocardial disturbances in diabetes either by an enhanced formation of angiotensin II or an accelerated degradation of bradykinin.

Acknowledgements. This work was supported by the Ministerium für Frauen, Familie und Gesundheit der Bundesrepublik Deutschland and the Wissenschaftsministerium des Landes NRW, the Deutsche Forschungsgemeinschaft (SFB 351), Bonn, and the "Klinische Zellbiologie und Biophysik" e.V., Düsseldorf, Germany.

\section{References}

1. Kannel WB, McGee FL (1979) Diabetes and cardiovascular disease: the Framingham study. JAMA 241: 2035 2038

2. Palumbo PJ, Elveback CR, Conolly DC (1981) Coronary artery disease and congestive heart failure in the diabetic: epidemiological aspects: The Rochester Project. In: Scott $\mathrm{RC}$ (ed) Clinical cardiology and diabetes. pp 13-34

3. Uusitupa MIJ, Mustonen JN, Airaksinen KE (1990) Diabetic heart muscle disease. Ann Med 22: 377-386

4. Rösen P, Pogatsa G, Tschöpe D, Addicks K, Reinauer H (1992) Diabetische Kardiopathie: Pathophysiologische Konzepte und therapeutische Ansätze. Klin Wochenschr. 69: 3-15

5. Zoneraich S (1988) Small vessel disease, coronary vasodilation reserve, and diabetic microangiopathy. Chest 94: 5-7

6. Cannon RO, Cunnion RE, Parillo JE et al. (1987) Dynamic limitation of coronary vasodilator reserve in patients with dilated cardio-myopathy and chest pain. J Am Coll Cardiol 10: $1190-1200$

7. Fein FS, Sonnenblick EH (1985) Diabetic cardiomyopathy. Prog Cardiovasc Dis 27: 255-270

8. Rösen P, Windeck P, Zimmer HG, Frenzel H, Burrig KF, Reinauer H (1986) Myocardial performance and metabolism in non-ketotic, diabetic rat hearts: myocardial function and metabolism in vivo and in the isolated perfused heart under the influence of insulin and octanoate. Basic Res Cardiol 81: 620-635

9. Rösen P, Kiesel U, Reinauer H, Boy C, Addicks K (1991) Cardiopathy in the spontaneously diabetic (BB)rat: evidence for microangiopathy and autonomic neuropathy in the diabetic heart.In: Nagano M, Dhalla NS (eds) The diabetic heart. Kluver Press, New York, pp 145-157

10. Rodrigues B, McNeill JH (1992) The diabetic heart: metabolic causes for the development of a cardiomyopathy. Cardiovasc Res 26: 913-922

11. Bhimji S, Godin DV, McNeill JH (1986) Insulin reversal of biochemical changes in hearts from diabetic rats. Am J Physiol 251: H670-H675

12. Regan TJ, Lyons MM, Ahmed SS (1977) Evidence for cardiomyopathy in familial diabetes mellitus. J Clin Invest 60 : 885-889

13. Hamby RI, Zoneraich S, Shermann L (1974) Diabetic cardiomyopathy. JAMA 229: 1749-1754

14. Shapiro LM (1985) Diabetes-induced heart muscle disease and left ventricular dysfunction. Practical Cardiol 11: 79--91

15. Koltai MZ, Rösen P, Hadhazy P, Ballagi-Pordany G, Koszeghy A, Pogatsa G (1988) Effects of hypoxia and adrenergic stimulation induced alterations in PGI2 synthesis by diabetic coronary arteries. J Diabetic Complications 2: 5-7

16. Tahiliani AG, McNeill JH (1986) Diabetes-induced abnormalities in the myocardium. Life Sci 38: 959-974 
17. Rösen P, Rösen R, Hohl C, Reinauer H, Klaus W (1984) Reduced transcoronary exchange and prostaglandin synthesis in diabetes rat heart. Am J Physiol 247: H563-H569

18. Addicks K, Boy C, Rösen P (1993) Sympathetic autonomic neuropathy in the heart of the spontaneous diabetic BB rat. Annals of Anatomy - Anatomischer Anzeiger 175: 253257

19. Regan TJ, Ettinger PO, Khan MI et al. (1974) Altered myocardial function and metabolism in chronic diabetes mellitus without ischemia in dogs. Circ Res 35: 222-237

20. Pierce GN, Dhalla NS (1985) Mechanisms of the defect in cardiac myofibrillar function during diabetes. Am J Physiol 248: E170-E175

21. Dominiak P (1993) Modulation of sympathetic control by ACE inhibitors. Eur Heart J 14: 169-172

22. Meisel AS, Phillips C, Michel MC, Ziegler MG, Carter SM (1989) Regulation of cardiac $\beta$-adrenergic receptors by captopril. Circ 80: 669-675

23. Lindpaitner K, Ganten D (1991) The cardiac renin-angiotensin system. An appraisal of present experimental and clinical evidence. Circ Res 68: 905-921

24. Dostal DE, Baker KM (1993) Evidence for a role of an intracardic renin-angiotensin system in normal and failing heart. TCM 3: 67-74

25. Weber K, Brilla CG (1991) Pathological hypertrophy and cardiac interstitium. Fibrosis and renin-angiotensin-aldosterone system. Circ 83: 1849-1865

26. Dietz R, Haberbosch W, Osterziel KJ, Förderer T, Busch C (1992) Kardioreparative Effekte der ACE-Hemmer. Klin Wochenschr 69 [Suppl] (XXIX): 16-24

27. Clozel M, Kuhn H, Hefti F, Baumgartner HR (1991). Endothelial dysfunction and sub-endothelial monocyte macrophages in hypertension. Effect of angiotensin enzyme inhibition. Hypertension 18: 132-141

28. Rösen P, Eckel J, Reinauer H (1983) Influence of bradykinin on glucose uptake and metabolism studied in isolated cardiac myocytes and isolated perfused rat hearts. J Biol Chem 364: 1431-1438

29. Rett K, Jauch KW, Wicklmayr M, Dietze G, Fink E, Mehnert H (1986) Angiotensin converting enzyme inhibitors in diabetes: experimental and human experience. Postgrad Med J 62: 59-64

30. Jauch KW, Hartl W, Guenther B, Rett K, Dietze G (1987) Captopril enhances insulin responsiveness of forearm muscle tissue in non-insulin dependent diabetes mellitus. Eur J Clin Invest 17: 448-457

31. Cushman DW, Cheung HS (1971) Spectrophotometric assay and properties of the angiotensin converting enzyme of rabbit lung. Biochem Pharmacol 20: 1637-1648

32. Rösen R, Beck E, Rösen P (1988) Early vascular alterations in the diabetic heart. Acta Physiol Hun'g 72: 3-11

33. Hartmann JF, Szemplinski M, Hayes NS, Keegan ME, Slater EE (1988) Effects of the angiotensin enzyme inhibitor, lisinopril, on normal and diabetic rats. J Hypertension 6: $677-683$
34. Valentovic M, Elliott C (1986) Angiotensin converting enzyme activity as function of the duration of the streptozotocin (STZ)-induced diabetes. Fed Proc 45: 463

35. Toop M, Dallinger K, Jennings P, Barnett A (1986) Angiotensin converting enzyme (ACE): relationship to insulindependent diabetes and microangiopathy. Diabet Med 3: 455-457

36. Boy C, Rösen P, Bloch W, Hess A, Happich D, Addicks K (1993) Diabetic cardiopathy in the spontaneous diabetic BB rat is diminished by long term application of the angiotensin converting enzyme inhibitor captopril. Proc 3rd Int Symp on ACE (in press)

37. Raya TE, Gay RG, Aguirre M, Goldman S (1989) Importance of venodilation in the prevention of left ventricular dilatation after chronic large myocardial infarction in rats: a comparison of captopril and hydralazine. Circ Res 64 : 330-337

38. Jackson CV, McGrath GM, Tahiliani AG, Vadlamudi RV, McNeill JH (1985) A functional and ultrastructural analysis of experimental diabetic rat myocardium. Manifestation of a cardiomyopathy. Diabetes 34: 876-883

39. McDowell TS, Chapleau MW, Hajduzcok G, Abboud FM (1994). Baroreflex dysfunction in diabetes mellitus. I. Selective impairment of parasympathetic control of heart rate. Am J Physiol 266: H235-H243

40. McDowell TS, Chapleau MW, Hajduzcok G, Abboud PM (1994). Baroreflex dysfunction in diabetes mellitus. II. Site of baroreflex impairment in diabetic rabbits. Am J Physiol 266: H244-H249

41. Fein FS, Miller-Green B, Sonnenblick EH (1985) Altered myocardial mechanics in diabetic rabbits. Am J Physiol 248: H729-H736

42. Ward WF, Molteni A, Tsao Ch, Hinz JM (1990) Captopril reduces collagen and mast cell accumulation in irradiated rat lung. Int J Radiation Oncol 19: 1405-1409

43. Lindpaitner K, Jim MW, Niedermaier N, Wilhelm MJ, Ganten D (1990) Cardiac angiotensinogen and its local activation in the isolated perfused beating heart. Circ Res 67: 564-573

44. Yamaji T, Fakuhara T, Kinoshita M (1993) Increased capillary permeability to albumin in diabetic rat myocardium. Circ Res 72: 947-957

45. Wiemer G, Schölkens BA, Becker RHA, Busse R (1991) Ramiprilat enhances endothelial autocoid formation by inhibiting breakdown of endothelium-derived bradykinin. Hypertension 18: 558-563

46. Raya TE, Lee RW, Westhoff T, Goldman S (1989) Captopril restores hemodynamic responsiveness to atrial natriuretic peptide in rats with heart failure. Circ 80: 18861892

47. Baker KM (1991) Cardiac actions of angiotensin. J Vasc Med Biol 3: 30-37

48. Peach MJ (1977) Renin-angiotensin systems: biochemistry and mechanisms of action. Physiol Rev 57: $317-370$ 